\title{
Helium Nanodroplet Isolation Spectroscopy in an Undergraduate Teaching Laboratory
}

\author{
Paul L. Raston \\ Department of Chemistry and Biochemistry, James Madison University \\ Harrisonburg, Virginia 22807, USA
}

\begin{abstract}
A home-built helium nanodroplet isolation spectrometer has been utilized by undergraduate students in course-based experiments to investigate the rovibrational dynamics of small molecules. Helium nanodroplets are well known to simplify the spectroscopy of embedded molecules owing to their low temperature $(0.4 \mathrm{~K})$ and weakly interacting nature. In the infrared spectral region, this results in a small number of rotationally resolved lines that can often be collected and analyzed in several lab periods. We demonstrate the advantages of using this technique in an upper-level undergraduate chemistry course for which the laser spectroscopy of helium solvated ${ }^{13} \mathrm{C}$-labelled formic acid was investigated for the first time.
\end{abstract}

\section{INTRODUCTION}

Studying the medium/high resolution infrared spectroscopy of molecules in the gas phase is a standard physical chemistry experiment that is typically introduced early on in a laboratory course. For the most part, small linear molecules are investigated, with the most common being $\mathrm{HCl} ;{ }^{1}$ this is on account of its lightness, large transition dipole moment, and putative simplicity (effectively having only one unique rotational constant; inversely proportional to the moment of inertia, I). Typically, the goal of such an experiment is to perform a fit of spectroscopic parameters (ground and excited rotational constants, band origin, ...) to the line positions, ultimately affording the structural parameters of the molecule; the most rewarding one of which is typically the bond length, $r$ (as gotten from the relation $I=\mu r^{2}$ ). More complicated species with two unique moments of inertia are sometimes investigated in an undergraduate course, such as $\mathrm{CH}_{3} \mathrm{I}^{2}$ This naturally requires the inclusion of additional parameters in the fit, such as multiple band origins, rotational constants, and Coriolis coupling constants. The most complicated type of molecule having three unique moments of inertia is typically avoided in undergraduate spectroscopy labs because the infrared spectra are exceptionally rich, often displaying 1000s of lines at room temperature. 
The rotationally resolved spectrum of a given molecule can be simplified by lowering the rotational temperature of the sample. Various techniques can be used for this purpose, for example, buffer gas cooling, ${ }^{3}$ and cooling in a supersonic expansion. ${ }^{4}$ These techniques typically result in a reduction in the (rotational) temperature by one-two orders of magnitude, resulting in simplified spectra that are more feasible for students to perform an in-course analysis on. Such spectra, however, still contain many lines that necessitate a somewhat involved analysis: for example, the $12 \mathrm{~K}$ spectrum of formic acid in the $\mathrm{C}-\mathrm{O}$ stretching band ( $\sim 50 \mathrm{~cm}^{-1}$ broad) was found to contain $\sim 200$ lines, ${ }^{5}$ some of which are finely split due to the asymmetry of the molecule (i.e., "asymmetry splittings"). The rotationally resolved spectra can, however, be simplified even further by reducing the temperature by three orders of magnitude relative to the gas phase; this can be accomplished by embedding the molecule of interest in superfluid helium nanodroplets. ${ }^{6-8}$

The first high-resolution investigation of a molecule in superfluid helium nanodroplets was performed on $\mathrm{SF}_{6}$, for which the infrared spectrum revealed rotationally resolved substructure that is consistent with a rotational temperature of $\sim 0.4 \mathrm{~K} .{ }^{9}$ Several years later the infrared helium nanodroplet spectrum of OCS in the C-O stretching fundamental was reported, ${ }^{10}$ which contains a small number of baseline resolved $P$ and $R$ branch peaks that can be analyzed using standard linear rotor energy level expressions. A couple of years later, formic acid was investigated in the vicinity of the $\mathrm{O}-\mathrm{H}$ and $\mathrm{C}-\mathrm{H}$ stretching fundamentals. ${ }^{11}$ While evidence for extensive Fermi and Coriolis coupling was found (due to differing linewidths between the bands), the rotational substructure within each band was well resolved, and analysis of the small number of peaks $(\leq 6)$ within each band resulted in determination of the rovibrational parameters $\left(v_{o}, A, B, C\right)$. In the following, we describe the value of employing this technique in an undergraduate teaching lab, and show data (and analysis) collected (and performed) by students on formic acid in helium nanodroplets.

\section{THE EXPERIMENT}

The helium nanodroplet isolation spectrometer the experiments were performed on was mostly built by students over the course of $\sim 2$ years. It has been described in detail elsewhere, 12-13 and here we provide only pertinent details; for additional details of the technique please see Refs. 6-8. Figure 1 shows the spectrometer, highlighting the three vacuum chambers that are labelled according to their functionality: the "Source Chamber", "Pick-up Chamber", and 
"Detection Chamber". Helium nanodroplets are produced in the source chamber by expanding ultra-pure carrier grade $(99.9995 \%)$ helium at a pressure of 34.5 bar through a nozzle $(\sim 5 \mu \mathrm{m})$ held at $\sim 19.5 \mathrm{~K}$. Under these conditions the average droplet size $(<N>)$ is $2.4 \times 10^{3}$ atoms, which is determined using well established empirical scaling laws that relate it to the backing pressure, nozzle size, and nozzle temperature. ${ }^{14}$ The expansion is skimmed ( $0.51 \mathrm{~mm}$ dia.) at the end of the source chamber, and the newly formed helium droplet beam passes into the pick-up chamber where it encounters gas phase ${ }^{13} \mathrm{C}$-labelled formic acid. The pressure of the dopant is optimized so that on average, one molecule is picked-up per droplet. The pick-up process involves a room temperature molecule colliding with a helium nanodroplet; the thermal conductivity of superfluid helium is $30 \mathrm{x}$ that of copper, and this allows for the excess kinetic energy of the molecule to be rapidly liberated through the evaporation of several hundred helium atoms. The captured molecule at $0.4 \mathrm{~K}$ can then be interrogated by laser spectroscopy before entering the detection chamber (vide infra).

The output of an external cavity quantum cascade laser $(\sim 9-10 \mu \mathrm{m})$ was aligned antiparallel to the helium nanodroplet beam. It was chopped and focused to a point somewhere in between pick-up and detection, and can be scanned with a modified program previously developed. ${ }^{15}$ When the infrared laser beam is resonant with a rovibrational transition of a captured formic acid molecule, it can absorb a photon. In the $\mathrm{C}-\mathrm{O}$ stretching band investigated here this corresponds to an energy $\left(E_{n} / h c\right)$ of $\sim 1095 \mathrm{~cm}^{-1}$, and since the binding energy of helium is $\sim 5 \mathrm{~cm}^{-1},{ }^{16}$ absorption of a photon will (on paper) result in the evaporation of 219 atoms from the droplet (i.e., $\Delta N=1095 / 5$ ). After the droplet beam is interrogated by the output of the laser, it encounters an off-axis quadrupole mass spectrometer that is housed in the detection chamber. A small fraction of droplets are ionized by electron impact in this chamber, with a probability that is proportional to its geometric cross section (which varies by $N^{2 / 3}$ ). Ionization of doped droplets typically gives rise to a positive charge on helium that migrates through the droplet until it encounters the embedded molecule (such as $\mathrm{H}^{13} \mathrm{COOH}$ ), and since the ionization potential of any impurity is substantially less than that of helium, droplet explosion and fragmentation typically takes place. ${ }^{17}$ Resulting positively charged fragments with masses greater than $\sim 6 \mathrm{u}$ were deflected through the quadrupole mass filter and detected with an electron multiplier tube. The resulting signal is processed with a lock-in amplifier with a phase angle optimized such that a positive output signal corresponds to depletion. 


\section{INSTRUMENT PREPARATION AND DATA ACQUISITION}

Helium nanodroplet isolation spectrometers consist of a large number of components, some of which are permanently on while others are on for the duration of the experiment. Major equipment that falls into the latter category on the JMU helium nanodroplet isolation spectrometer are the diffusion pumps, closed cycle helium cryostat, and mass spectrometer (see figure 1). Our system is configured such that we can remotely activate these components by way of an email to a Raspberry $\mathrm{Pi}^{\mathrm{TM}}$. We turn these components on a couple of hours before experimentation, with the cool down of the coldhead taking the longest. Students enter the lab with the spectrometer after this time, and configure it for the planned experiment. This involves transferring several $\mathrm{mL}$ of liquid sample (for the formic acid experiment) into a round bottom flask with $1 / 4$ " SS pipe fitting, and coupling that to an inlet flange on the pickup chamber. A needle valve is used to control the sample flow into the chamber, and after evacuating the head gas, it is adjusted to a pressure of $\sim 5 \times 10^{6}$ torr, ${ }^{18}$ which is optimized for the pick-up of one molecule per droplet. It is instructive to measure the mass spectrum of the neat droplet beam, and compare it with what is observed after doping the droplets. Before doing this, students fill the liquid nitrogen Dewar's (on the pick-up and detection chambers) and open a solenoid driven pneumatic gate valve (8" CF) that separates that last two chambers.

After establishing that helium droplet conditions are optimized, students turn on "optical" elements, which correspond to the infrared laser, piezo driver, wavemeter, and chopper wheel. Before scanning the laser, the group of students discuss a wavenumber range that would be most efficient to scan based on previous helium droplet investigations (which show vibrational shifts in going from the gas phase to helium is $\sim 1 \mathrm{~cm}^{-1}$ ), and if available, the band origin, band type, and rotational constants of the target molecule in the gas phase. For $\mathrm{H}^{13} \mathrm{COOH}$ in the $\mathrm{C}-\mathrm{O}$ stretching band, these are very accurately known. ${ }^{19}$ After establishing suitable parameters for collecting the spectrum (scan range, scan speed, data acquisition rate, lock-in sensitivity...), students begin scanning and acquiring data. While we have LabVIEW programs that fully automate the scanning, it more engaging to manually control the coarse stepping of the laser (while a separate program applies a triangle wave to the piezo element for the fine scanning). In this way, over the course of about two hours, the data corresponding to the spectrum shown in figure 2 was collected (this is after data reduction ${ }^{15}$ ). 


\section{SPECTROSCOPIC ANALYSIS}

\section{Computer Assisted Fitting}

A number computer programs have been developed that can be utilized for fitting spectroscopic parameters to rotationally resolved spectra. ${ }^{20-26}$ Arguably, the most user friendly of these is PGOPHER, ${ }^{24}$ and this makes it well suited for incorporation into undergraduate coursework. The spectrum shown in figure 2 was collected by one group of six students who were enrolled in the Biophysical Chemistry course, each of which performed a fit of the spectroscopic parameters to the line positions. Leading into this we had covered rovibrational spectroscopy of nothing more complicated than linear molecules in our guided inquiry Quantum Chemistry \& Spectroscopy course (that all six students were enrolled in). Additional background material on asymmetric tops $\left(I_{a}=I_{b}=I_{c}\right)$ had to be covered to ensure that the significance of the molecular parameters used in the model were adequately understood.

Students began by simulating the infrared spectrum of gas phase $\mathrm{H}^{13} \mathrm{COOH}$ with PGOPHER, using the known band origin and rotational constants (ground and excited $\mathrm{C}-\mathrm{O}$ stretching states), ${ }^{19,27}$ along with a reasonable estimate of the band type (which can be readily done with most electronic structure computational packages). Although they did not include the higher order (centrifugal distortion and Coriolis coupling) constants, an interesting extension of this investigation would be to record the gas phase spectrum of $\mathrm{H}^{13} \mathrm{COOH}$ and then simulate it. Since the simulated temperate is $300 \mathrm{~K}$ (by default), 100s of rotational states are populated which gives rise to 1000 s of lines. An extended activity on the Boltzmann distribution ("Population of Quantum States"28) was covered before fitting the spectrum, which gave students the necessary background on the effect that temperature has on the infrared band profiles. Thus the very dramatic effect that reducing the temperature in the simulation from $300 \mathrm{~K}$ to $0.4 \mathrm{~K}$ (the temperature of helium droplets) was immediately appreciated. In fact, at this stage the simulated lines can be readily assigned to the observed ones, and the spectroscopic parameters can be optimized. The result of doing this is shown in figure 2, which shows excellent agreement between experiment and theory.

\section{Manual Fitting}

An alternative method of analyzing the spectrum is to determine the constants by taking the combination differences of transitions. This method requires more of an understanding of asymmetric top molecular energy levels so that assignments can be readily made (see figure 
1). The first step is to generate a list of line positions, assignments, and energy level expressions ${ }^{29}$ similar to that shown in table 2 , within which we label each transition with a lowercase italicized letter (for convenience). The logical starting point in analyzing the spectrum shown in figure 1 seems to lie in taking the difference between transition $f$ and $e$,

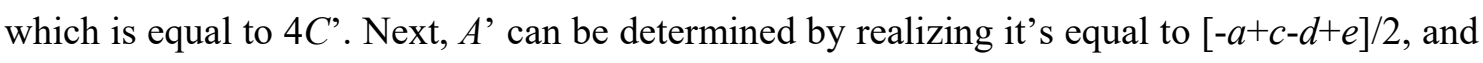
similarly, $B^{\prime}$ is equal to $[a-c-d+e] / 2$. The band origin can then be determined by subtracting the appropriate (just determined) upper state rotational constants from either transition $a$ or $c$. Finally, $B$ " $+C$ " is most easily gotten by subtracting $d$ from the band origin. It is satisfying to perform a check to see if the constants are correct by calculating transition $b$ (the only one not included in the analysis) using all of the spectroscopic constants.

\section{Hybrid Approach}

What we label as the "hybrid approach" involves constructing table 2 (i.e., assigning peaks and determining term values), then performing a least-squares fit using a computer. While a number of different programming languages can be used for this (e.g., FORTRAN, C++, Mathematica...), we opted for Python (with the NumPy and SciPy extension modules). The code we used (see ) could be developed by students with little programming experience in the time taken to average the spectra ( $\sim 2$ hours). It returns the same constants as did PGOPHER (see table 1).

\section{INERTIAL ANALYSIS}

Following the spectroscopic analysis, an inertial analysis allows for a closer connection to be made with the physical attributes of the system. The moment of inertia about a certain axis is obtained by inverting the corresponding rotational constant, e.g., $I_{a}=\frac{h}{8 \cdot \pi^{2} \cdot c \cdot m_{u} \cdot A}$ where $I_{a}$ is the moment of inertia about the $a$-axis (in amu $\cdot \mathrm{m}^{2}$ ), $h$ is Planck's constant (in $\mathrm{J} \cdot \mathrm{s}$ ), $m_{u}$ is the atomic mass constant (in $\mathrm{kg} / \mathrm{amu}$ ), $c$ is the speed of light (in $\mathrm{cm} / \mathrm{s}$ ), and $A$ is the rotational constant about the $a$-axis (in $\mathrm{cm}^{-1}$ ). A convenient conversion ratio (if pressed for time) is $I_{a}=\left(16.8576 \cdot \mathrm{amu} \cdot \AA^{2} \cdot \mathrm{cm}^{-1}\right) / A$. In this way the moments of inertia about the three inertial axes for $\mathrm{H}^{13} \mathrm{COOH}$ were obtained, both in the gas phase and in superfluid helium nanodroplets (see table 3).

By taking the difference between the gas phase and helium solvated moments of inertia, one obtains the moment of inertia of helium $\left(\Delta I_{\mathrm{He}}\right)$ that couples to each of the molecular axes. 
What the students found for this molecule is that the amount of helium that couples to rotation decreases with increasing angular velocity (which is proportional to the rotational constant; see table 3): as noted by one team of students, "The moment of inertia for helium about the $a$-axis may be concerningly low, however, since the rotational constant is so high for the $a$-axis, it may be explained that since formic acid is rotating so fast, it may not drag as much helium along for the ride." This is known as a breakdown of the adiabatic following approximation, and reference to the literature on this aspect of the investigation can be made. ${ }^{12-13,30-31}$

\section{SUMMARY \& OUTLOOK}

Here we highlighted the value of including the technique of helium nanodroplet isolation spectroscopy in a teaching laboratory by way of an example molecule that was explored by undergraduate students. The molecule chosen was $\mathrm{H}^{13} \mathrm{COOH}$ because it is biophysically relevant, it has large C-O stretching transition moment, it is relatively small (which allows for rotationally resolved peaks), and most importantly is accessible with our (borrowed) laser system. This investigation was performed after analyzing the infrared spectra of the linear rotors, $\mathrm{CO}$ and $\mathrm{CO}_{2}$ (formed from the pyrolysis of formic acid), in addition to covering basic molecular spectroscopy in an activity-based Quantum Chemistry \& Spectroscopy course. The primary learning outcome was that students got to advance their knowledge of rovibrational spectroscopy by investigating an asymmetric rotor, and in doing so they utilized state-of-theart instrumentation that is uncommonly included in undergraduate courses.

It is important to note that while the normal isotopologue of formic acid has previously been explored in helium nanodroplets (as has the monodeuterated form), ${ }^{11,32}$ this is the first time that ${ }^{13} \mathrm{C}$-labelled formic acid has been investigated. Part of the motivation in doing so was to provide a mini course-based undergraduate research experience (CURE), ${ }^{33}$ with the intention of having student(s) continue the research project after completion of the course it was seeded in. Future research directions include investigating the infrared spectra of formic acid clusters, beyond the dimer, ${ }^{34}$ and on this end a spectrum has been collected in-lab with preliminary assignments made (see figure S1). In closing, we note that there are many potentially interesting unexplored molecules that can be investigated by helium nanodroplet isolation spectroscopy, and a relatively simple one we plan to investigate in 
Physical Chemistry II Lab is methyl fluoride $\left(\mathrm{CH}_{3} \mathrm{~F}\right)$, since it is small, is compatible with our current laser system, and has a very bright C-F stretching vibration.

Table 1. Spectroscopic parameters of $\mathrm{H}^{13} \mathrm{COOH}$ embedded in helium nanodroplets; uncertainties (in last place) are estimated. Values were determined by students in biophysical chemistry course using PGOPHER.

\begin{tabular}{c|ccccc}
\hline & $v_{o}$ & $A^{\prime}$ & $B^{\prime}$ & $C^{\prime}$ & $\left(B^{\prime \prime}+\mathrm{C}^{\prime \prime}\right) / 2$ \\
\hline $\mathrm{Gas}^{\mathrm{a}}$ & 1095.403646 & 2.522449 & 0.40012796 & 0.34396941 & 0.37413491 \\
$\mathrm{He}$ & $1094.954(2)$ & $1.451(2)$ & $0.268(1)$ & $0.206(1)$ & $0.254(1)$ \\
$\mathrm{Gas} / \mathrm{He}$ & 1.000411 & 1.739 & 1.492 & 1.668 & 1.473 \\
\hline
\end{tabular}

${ }^{\mathrm{a} F r o m ~ R e f . ~} 35$.

Table 2. Lines positions and assignments for $\mathrm{H}^{13} \mathrm{COOH}$ embedded in helium nanodroplets. Also included are the rigid asymmetric rotor energy level expressions for the ground (") and excited ( ) states.

\begin{tabular}{llllc}
\hline$J^{\prime} K a^{\prime} K c^{\prime}-J^{\prime \prime} K a^{\prime \prime} K c^{\prime \prime}$ & $v\left(\mathrm{~cm}^{-1}\right)$ & $F^{\prime}$ & $F^{\prime \prime}$ & label \\
\hline $1_{01-0_{00}}$ & 1095.420 & $B^{\prime}+C^{\prime}$ & 0 & $a$ \\
$2_{02}-1_{01}$ & 1095.873 & $2 A^{\prime}+2 B^{\prime}+2 C^{\prime}-2\left(\left(B^{\prime}-C^{\prime}\right)^{2}\right.$ & $B^{\prime \prime}+C^{\prime \prime}$ & $b$ \\
& & & \\
$1_{11-0}-0_{00}$ & 1096.620 & $\left.\left.A^{\prime}+C^{\prime}-C^{\prime}\right)\left(A^{\prime}-B^{\prime}\right)\right)^{1 / 2}$ & 0 & $c$ \\
$0_{00}-1_{01}$ & 1094.449 & 0 & $B^{\prime \prime}+C^{\prime \prime}$ & $d$ \\
$1_{10-1}$ & 1096.161 & $A^{\prime}+B^{\prime}$ & $B^{\prime \prime}+C^{\prime \prime}$ & $e$ \\
$2_{12-1}$ & 1096.985 & $A^{\prime}+B^{\prime}+4 C^{\prime}$ & $B^{\prime \prime}+C^{\prime \prime}$ & $f$ \\
\hline
\end{tabular}

Table 3. Moments of inertia from helium nanodroplet experiment on $\mathrm{H}^{13} \mathrm{COOH}$ (values for the excited vibrational state). Units are amu* $\AA^{2}$.

${ }^{\mathrm{a} F r o m ~ R e f . ~} 35$.

\begin{tabular}{c|ccc}
\hline & He + rotor & rotor $^{\mathrm{a}}$ & $\mathrm{He}$ \\
\hline$I_{a}$ & 11.6 & 6.68 & 4.93 \\
$I_{b}$ & 62.9 & 42.1 & 20.8 \\
$I_{c}$ & 81.8 & 49.0 & 32.8 \\
\hline
\end{tabular}




\section{REFERENCES}

1. Stafford, F. E.; Holt, C. W.; Paulson, G. L., Vibration-rotation spectrum of HCl: A physical chemistry experiment. J. Chem. Ed. 1963, 40 (5), 245.

2. McNaught, I. J., Structural parameters of methyl iodide by infrared spectroscopy. $J$. Chem. Ed. 1982, 59 (10), 879.

3. Messer, J. K.; De Lucia, F. C., Measurement of Pressure-Broadening Parameters for the CO-He System at 4 K. Phys. Rev. Lett. 1984, 53 (27), 2555-2558.

4. Smalley, R. E.; Ramakrishna, B. L.; Levy, D. H.; Wharton, L., Laser spectroscopy of supersonic molecular beams: Application to the $\mathrm{NO}_{2}$ spectrum. J. Chem. Phys. 1974, 61 (10), 4363-4364.

5. Goroya, K. G.; Zhu, Y.; Sun, P.; Duan, C., High resolution jet-cooled infrared absorption spectra of the formic acid dimer: A reinvestigation of the $\mathrm{C}-\mathrm{O}$ stretch region. $J$. Chem. Phys. 2014, 140 (16), 164311.

6. Toennies, J. P.; Vilesov, A. F., Superfluid Helium Droplets: A Uniquely Cold Nanomatrix for Molecules and Molecular Complexes. Angew. Chem.-Int. Edit. 2004, 43 (20), 2622-2648.

7. Stienkemeier, F.; Lehmann, K. K., Spectroscopy and Dynamics in Helium Nanodroplets. Journal of Physics B: Atomic, Molecular and Optical Physics 2006, 39 (8), R127-R166.

8. Choi, M. Y.; Douberly, G. E.; Falconer, T. M.; Lewis, W. K.; Lindsay, C. M.; Merritt, J. M.; Stiles, P. L.; Miller, R. E., Infrared Spectroscopy of Helium Nanodroplets: Novel Methods for Physics and Chemistry. Int. Rev. Phys. Chem. 2006, 25 (1-2), 15-75.

9. Hartmann, M.; Miller, R. E.; Toennies, J. P.; Vilesov, A., Rotationally Resolved Spectroscopy of $\mathrm{SF}_{6}$ in Liquid-Helium Clusters - a Molecular Probe of Cluster Temperature. Phys. Rev. Lett. 1995, 75 (8), 1566-1569.

10. Grebenev, S.; Hartmann, M.; Havenith, M.; Sartakov, B.; Toennies, J. P.; Vilesov, A. F., The rotational spectrum of single OCS molecules in liquid ${ }^{4} \mathrm{He}$ droplets. $J$. Chem. Phys. 2000, 112 (10), 4485-4495.

11. Madeja, F.; Markwick, P.; Havenith, M.; Nauta, K.; Miller, R. E., Rotationally resolved infrared spectroscopy of $\mathrm{h}_{2}$ and $\mathrm{d}_{1}$ formic acid monomer in liquid He droplets. $J$. Chem. Phys. 2002, 116 (7), 2870-2878.

12. Faulkner, T.; Miller, I.; Raston, P. L., Quantum Cascade Laser Spectroscopy of OCS Isotopologues in ${ }^{4} \mathrm{He}$ Nanodroplets: A Test of Adiabatic Following for a Heavy Rotor. $J$. Chem. Phys. 2018, 148 (4), 044308. 
13. Miller, I.; Faulkner, T.; Raston, P. L., Laser Spectroscopy of Methanol Isotopologues in ${ }^{4} \mathrm{He}$ Nanodroplets: Probing the Inertial Response around a Moderately Light Rotor. $J$. Phys. Chem. A 2019, 123 (8), 1630-1636.

14. Knuth, E.; Schilling, B.; Toennies, J. P., Proceedings of the $19^{\text {th }}$ International Symposium on Rarefied Gas Dynamics. Oxford University Press: London, 1995.

15. Morrison, A. M.; Liang, T.; Douberly, G. E., Automation of an "Aculight" Continuous-Wave Optical Parametric Oscillator. Rev. Sci. Inst. 2013, 84 (1), 013102.

16. Pandharipande, V. R.; Zabolitzky, J. G.; Pieper, S. C.; Wiringa, R. B.; Helmbrecht, U., Calculations of Ground-State Properties of Liquid ${ }^{4}$ He Droplets. Phys. Rev. Lett. 1983, 50 (21), 1676-1679.

17. Lewis, W. K.; Applegate, B. E.; Sztáray, J.; Sztáray, B.; Baer, T.; Bemish, R. J.; Miller, R. E., Electron Impact Ionization in Helium Nanodroplets: Controlling Fragmentation by Active Cooling of Molecular Ions. J. Am. Chem. Soc. 2004, 126 (36), 11283-11292.

18. We note this is the raw (uncorrected) ion gauge reading.

19. Ong, P. P.; Goh, K. L.; Teo, H. H., Analysis of High-Resolution FTIR Spectrum of the v6 Band of $\mathrm{H}^{13} \mathrm{COOH}$. J. Mol. Spec. 1999, 194 (2), 203-205.

20. Pickett, H. M., The fitting and prediction of vibration-rotation spectra with spin interactions. J. Mol. Spec. 1991, 148 (2), 371-377.

21. Tasinato, N.; Charmet, A. P.; Stoppa, P., ATIRS package: A program suite for the rovibrational analysis of infrared spectra of asymmetric top molecules. J. Mol. Spec. 2007, 243 (2), 148-154.

22. Seifert, N. A.; Finneran, I. A.; Perez, C.; Zaleski, D. P.; Neill, J. L.; Steber, A. L.; Suenram, R. D.; Lesarri, A.; Shipman, S. T.; Pate, B. H., AUTOFIT, an automated fitting tool for broadband rotational spectra, and applications to 1-hexanal. J. Mol. Spec. 2015, 312, 1321.

23. Licari, D.; Tasinato, N.; Spada, L.; Puzzarini, C.; Barone, V., VMS-ROT: A New Module of the Virtual Multifrequency Spectrometer for Simulation, Interpretation, and Fitting of Rotational Spectra. J. Chem. Theory Comput. 2017, 13 (9), 4382-4396.

24. Western, C. M., PGOPHER: A program for simulating rotational, vibrational and electronic spectra. J. Quant. Spectrosc. Radiat. Transf. 2017, 186, 221-242.

25. Zaleski, D. P.; Prozument, K., Automated assignment of rotational spectra using artificial neural networks. J. Chem. Phys. 2018, 149 (10).

26. Western, C. M.; Billinghurst, B. E., Automatic and semi-automatic assignment and fitting of spectra with PGOPHER. Phys. Chem. Chem. Phys. 2019, 21 (26), 13986-13999. 
27. Willemot, E.; Dangoisse, D.; Monnanteuil, N.; Bellet, J., Microwave spectra of molecules of astrophysical interest. XVIII. Formic acid. J. Phys. Chem. Ref. Data 1980, 9 (1), 59-160.

28. Shepherd, T. D.; Grushow, A., Quantum Chemistry and Spectroscopy: A Guided Inquiry. Wiley: 2014.

29. P. F. Bernath, Spectra of Atoms and Molecules. 1995.

30. Conjusteau, A.; Callegari, C.; Reinhard, I.; Lehmann, K. K.; Scoles, G., Microwave Spectra of HCN and DCN in ${ }^{4} \mathrm{He}$ Nanodroplets: A Test of Adiabatic Following. J. Chem. Phys. 2000, 113 (12), 4840-4843.

31. Hoshina, H.; Skvortsov, D.; Sartakov, B. G.; Vilesov, A. F., Rotation of Methane and Silane Molecules in He Droplets. J. Chem. Phys. 2010, 132 (7), 074302.

32. Das, P.; Knapp, C. J.; Jäger, W., Ro-vibrational spectroscopy of the formic acid-d 1 monomer embedded in helium nanodroplets. J. Mol. Spec. 2017, 341, 17-22.

33. https://serc.carleton.edu/curenet/collection/221478.html.

34. Madeja, F.; Havenith, M.; Nauta, K.; Miller, R. E.; Chocholoušová, J.; Hobza, P., Polar isomer of formic acid dimers formed in helium nanodroplets. J. Chem. Phys. 2004, 120 (22), 10554-10560.

35. Baskakov, O.; Horneman, V. M.; Alanko, S.; Lohilahti, J., FTIR spectra of the $v_{6}$ and $v_{8}$ bands of ${ }^{13} \mathrm{C}$ formic acid molecule-Assignment of FIR-laser lines. J. Mol. Spec. 2008, 249 (1), 60-64. 


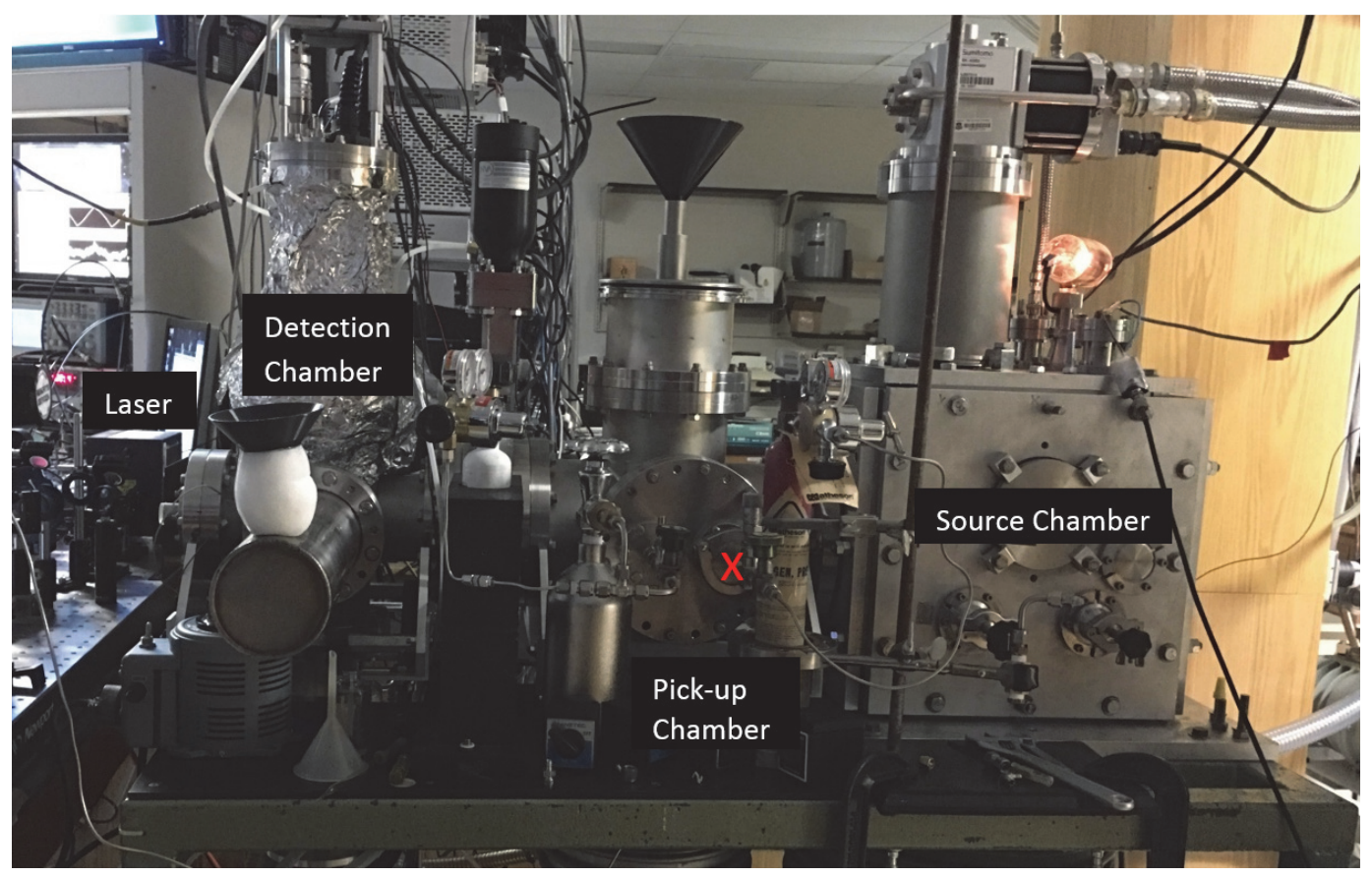

Figure 1. The JMU helium nanodroplet isolation spectrometer. The source and pick-up chambers are evacuated with diffusion pumps and the detection chamber is evacuated with a turbo pump. 


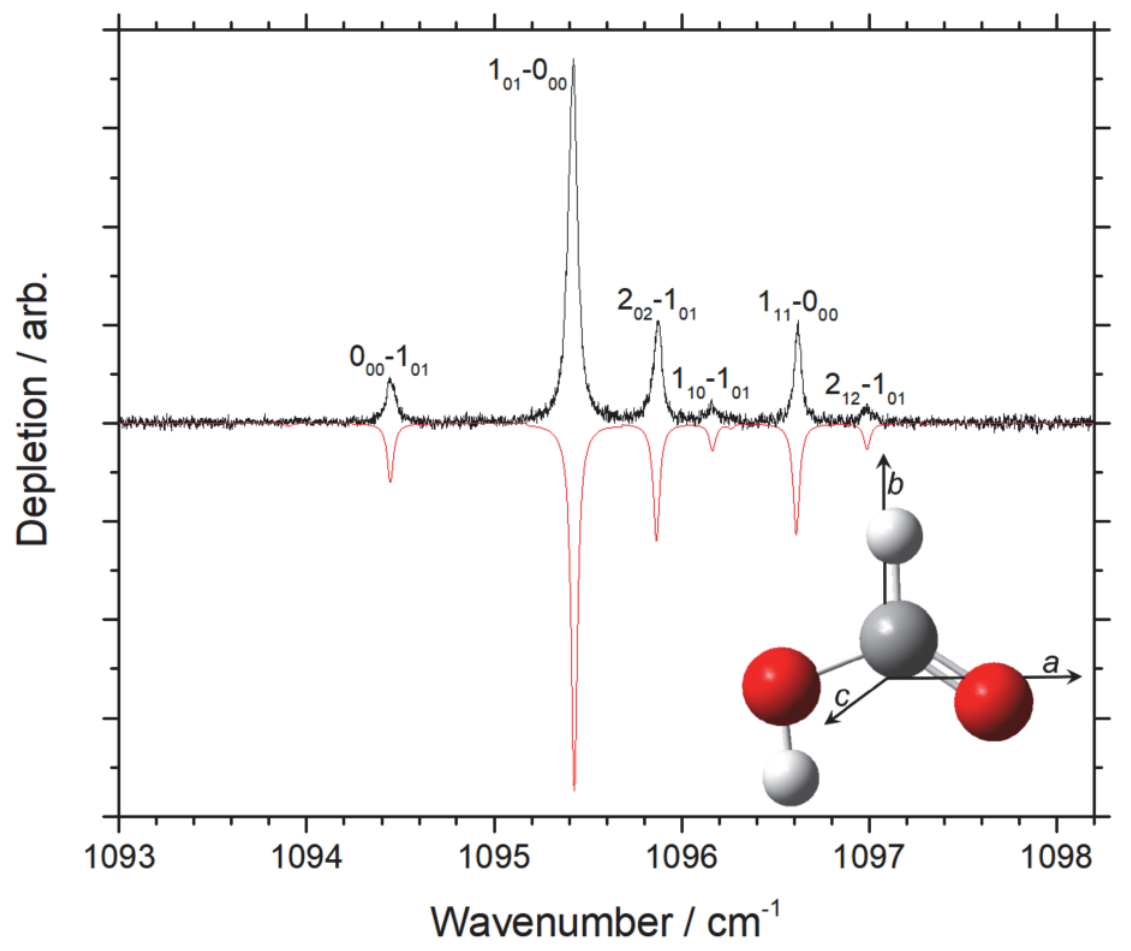

Figure 2. Experimental (black) and simulated (red) spectra of $\mathrm{H}^{13} \mathrm{COOH}$ in helium nanodroplets in the $\mathrm{C}-\mathrm{O}$ stretching fundamental. A temperature of $0.4 \mathrm{~K}$ and Lorentzian linewidth of $0.04 \mathrm{~cm}^{-1}$ was used in the simulation. The inset shows the principal axes superimposed on the optimized structure of formic acid. From the relative intensities of the $a$ and $b$ - type bands, the $\mu_{a}: \mu_{b}$ ratio was determined to be 1.8 . 